\title{
Expanding the Ig Superfamily Code for Laminar Specificity in Retina: Expression and Role of Contactins
}

\author{
Masahito Yamagata and Joshua R. Sanes \\ Department of Molecular and Cellular Biology and Center for Brain Science, Harvard University, Cambridge, Massachusetts 02138
}

\begin{abstract}
Bipolar, amacrine, and retinal ganglion cells elaborate arbors and form synapses within the inner plexiform layer (IPL) of the vertebrate retina. Specific subsets of these neuronal types synapse in one or a few of the $\geq 10$ sublaminae of the IPL. Four closely related Ig superfamily transmembrane adhesion molecules-Sidekick1 (Sdk1), Sdk2, Dscam, and DscamL-are expressed by non-overlapping subsets of chick retinal neurons and promote their lamina-specific arborization (Yamagata and Sanes, 2008). Here, we asked whether contactins (Cntns), six homologs of Sdks and Dscams, are expressed by and play roles in other subsets. In situ hybridization showed that cntn1-5 were differentially expressed by subsets of amacrine cells. Immunohistochemistry showed that each Cntn protein was concentrated in a subset of IPL sublaminae. To assess roles of Cntns in retinal development, we focused on Cntn2. Depletion of Cntn2 by RNA interference markedly reduced the ability of $\mathrm{Cntn} 2$-positive cells to restrict their arbors to appropriate sublaminae. Conversely, ectopic expression of cntn2 redirected neurites of transduced neurons to the Cntn2-positive sublaminae. Thus, both loss- and gain-of-function strategies implicate Cntn2 in lamina-specific neurite targeting. Studies in heterologous cells showed that Cntn2 mediates homophilic adhesion, but does not bind detectably to Sdks, Dscams, or other Cntns. Overexpression analysis showed that Cntns1 and 3 can also redirect neurites to appropriate sublaminae. We propose that Cntns, Sdks, and Dscams comprise an Ig superfamily code that uses homophilic interactions to promote lamina-specific targeting of retinal dendrites in IPL.
\end{abstract}

\section{Introduction}

The hallmark of the nervous system is the specificity of its synaptic connections. In the retina, on which we focus here, photoreceptors form synapses on interneurons that synapse in turn on retinal ganglion cells (RGCs); the RGCs then send information to the brain (Sanes and Zipursky, 2010). Whereas photoreceptors are essentially light detectors, RGCs respond selectively to visual features such as objects moving in a particular direction. The transformation from light detection to feature detection results from the specific connections made by $\sim 50$ types of interneurons (amacrine and bipolar cells) onto $\sim 25$ types of RGCs in a synaptic region called the inner plexiform layer (IPL) (Masland, 2001; Sanes and Zipursky, 2010; Wässle, 2004). The IPL is divided into 10 or more sublaminae (Roska and Werblin, 2001), with the arbors of each subtype being confined to just one or a few of these sublaminae. Thus, as elsewhere in the nervous system (Sanes and Yamagata, 2009), mechanisms that determine the laminae within which synaptic partners meet are major determinants of synaptic specificity.

In previous studies, we identified four closely related transmembrane Ig superfamily (IgSF) adhesion molecules-Sidekick-1

Received July 5, 2012; revised Aug. 11, 2012; accepted Aug. 21, 2012.

Author contributions: M.Y. and J.R.S. designed research; M.Y. performed research; M.Y. and J.R.S. analyzed data; M.Y. and J.R.S. wrote the paper.

This work was supported by NIH Grant R01 NS029169.

The authors declare no competing financial interests.

Correspondence should be addressed to Joshua R. Sanes, Center for Brain Science, Harvard University, 52 0xford Street Cambridge, MA 02138. E-mail: sanesj@mcb.harvard.edu.

DOI:10.1523/JNEUROSCI.3193-12.2012

Copyright $\odot 2012$ the authors $\quad 0270-6474 / 12 / 3214402-13 \$ 15.00 / 0$
(Sdk1), Sdk2, Dscam, and DscamL-that promote laminar specificity in chick retina (Yamagata et al., 2002; Yamagata and Sanes, 2008). They are expressed by mutually exclusive subsets of interneurons and RGCs in chick retina and neurons expressing each of the four send processes to a restricted subset of IPL sublaminae. In each case, presynaptic and postsynaptic neurons expressing the same gene arborize in the same sublaminae. Moreover, loss- and gain-of-function studies indicated that these IgSF proteins act to target or restrict processes to appropriate IPL sublaminae.

Sdks and Dscams together mark only around half of all retinal neurons, so other molecules are surely involved in promoting laminar specificity in the IPL. These molecules could be members of other families. Indeed, recent studies have shown critical roles for semaphorins and their receptors, the plexins, in this process (Matsuoka et al., 2011). In addition, other IgSF members might act in Sdk/Dscam-negative cells. Here, we analyzed contactins (Cntns) to test this possibility. The Cntns comprise a set of six homologous proteins: Cntn1/F3/F11, Cntn2/TAG-1/Axonin-1, Cntn3/BIG-1, Cntn4/BIG-2, Cntn5/NB2, and Cntn6/NB3. Cntns are the closest relatives of the Sdks and Dscams (Fig. 1) and all have been implicated in various aspects of neural development (for review, see Salzer et al., 2008; Shimoda and Watanabe, 2009; Stoeckli, 2010; Zuko et al., 2011). Thus, both their structure and their function encouraged us to test Cntns as mediators of laminar specificity.

We show here that cntns $1-5$ are expressed by subsets of retinal neurons and that the Cntn1-5 proteins are concentrated in distinct IPL sublaminae. Then, using Cntn2 as exemplar, we demonstrate that cntns promote lamina-specific targeting of neurites 

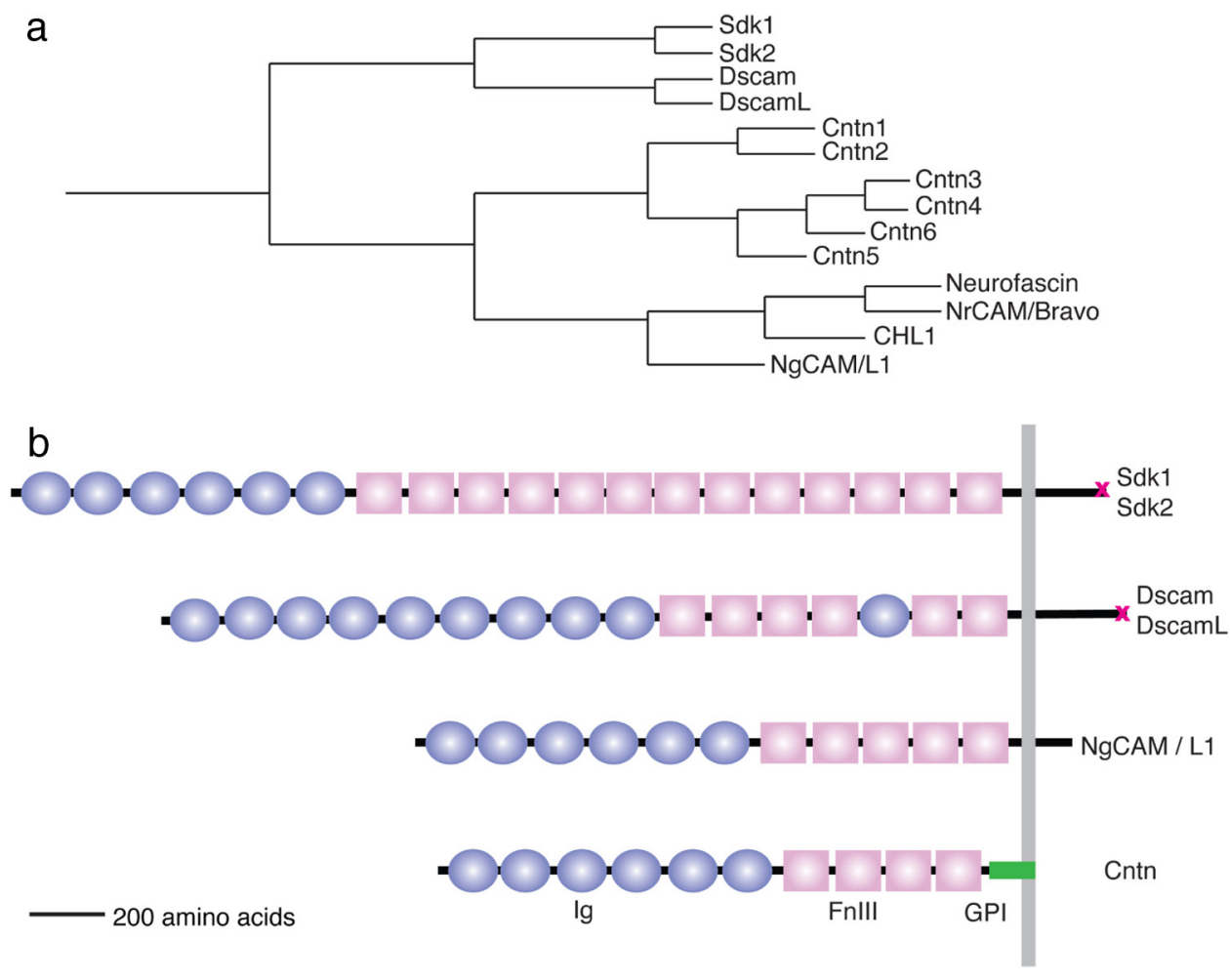

Figure 1. Sequence and structure of Contactins and related members of the IgSF subfamily. $\boldsymbol{a}$, Phylogenetic relationship of Cntns1- 6 and their closest relatives in the lgSF, the Sdks, Dscams, and $\mathrm{NgCAM} / \mathrm{L} 1 \mathrm{~s}$. Dendrogram was constructed using the Clustal $\omega$ algorithm. $\boldsymbol{b}$, Predicted domain structure of Cntns, Sdks, Dscams, and NgCAMs. Circles, Ig domains; rectangles, fibronectin type III (FnIII) domains; vertical line, plasma membrane; green bar, GPI anchor; X, PDZ domain-binding C-terminal sequence.

in the IPL. Together with previous results on Sdks and Dscams, these results suggest that a complex IgSF code regulates laminar specificity in retina.

\section{Materials and Methods}

Contactin sequence analysis and cDNAs. Clustal W2 analysis (http:// www.ebi.ac.uk/Tools/msa/clustalw2/) was used for initial comparison of $\sim 200$ IgSF molecules. Clustal $\omega$ (http://www.ebi.ac.uk/Tools/msa/ clustalo/) was used for detailed comparison of protein sequences (Sievers et al., 2011). Chick cntn cDNA sequences were obtained from GenBank.

Full-length chicken $c n t n 1,2,4$, and 5 cDNAs and partial chick $c n t n 3$ and $6 \mathrm{cDNAs}$ were amplified from oligo-dT-selected mRNA from embryonic day (E) 15 chick retina and optic tectum, using SuperScript III (Life Technologies), and Phusion high-fidelity thermostable DNA polymerase (New England BioLabs), and the products were cloned into pCR8-TOPO (Life Technologies). Because a full-length chick cntn3 sequence was not available in GenBank, we generated a cDNA encoding a chimeric protein by fusing $381 \mathrm{nt}$ of $5^{\prime}$ and $102 \mathrm{nt}$ of $3^{\prime}$ mouse cntn 3 sequence to the central $2601 \mathrm{nt}$ of chick cntn3.

In situ hybridization. For in situ hybridization, RNA probes were generated from linearized plasmids using T7 RNA polymerase (Life Technologies) and digoxygenin- or fluorescein-labeled nucleotides (Roche). In situ hybridization using nitro-blue tetrazolium and 5-bromo-4chloro-3'-indolyphosphate and double color in situ hybridization using TSA Plus (PerkinElmer) were performed as described by Yamagata et al. (2006), except that slides were incubated with $0.1 \mathrm{M}$ glycine- $\mathrm{HCl}, \mathrm{pH} 2.0$, for $30 \mathrm{~min}$ at room temperature following the first color reaction to remove peroxidase-conjugated anti-hapten antibodies and thereby avoid crossreactivity (Lauter et al., 2011).

Antibodies. A mouse monoclonal antibody to chick Cntn1 (clone F11, IgG1) was obtained from Fritz G. Rathjen (Max-Delbrück-Centrum, Berlin, Germany) (Rathjen et al., 1987). A mouse monoclonal antibody to chick Cntn2 (clone 23.4-5, IgG1) (Dodd et al., 1988) was obtained from Developmental Studies Hybridoma Bank. Mouse polyclonal antibodies to chick Cntns3, 4, and 5 were generated in mice by immunization with $c n t n$-transfected mouse L cells. An expression vector, pCAG-RfAIRES-neo, was constructed from pSNAP (New England Biolabs) by changing its eukaryotic promoter to CAG (CMV plus chick $\beta$-actin promoter) and replacing SNAP with Gateway cassette RfA (Life Technologies). This expression vector has a neomycin resistance gene downstream of an IRES (internal ribosome entry site) for selection of stable transfectants. Cntn cDNAs were transferred to the RfA site using LR clonase (Life Technologies). Mouse L fibroblasts (ATCC) were transfected using

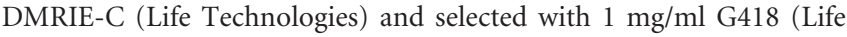
Technologies). Pooled stable transfectants were dissociated with trypsinEDTA (Life Technologies); the reaction was then stopped by adding fetal bovine serum-containing Dulbecco-F12 medium. Cells were rinsed with PBS three times, and resuspended in PBS. BALB/c female mice were injected intraperitoneally with $10^{7}$ cells in $0.5 \mathrm{ml} \mathrm{4-5}$ times at 2-3 weeks intervals, beginning at 6 weeks of age. Antiserum was then collected, incubated with paraformaldehyde-fixed untransfected L cells to remove irrelevant antibodies, and used for immunostaining.

To test the specificity of the anti-Cntn antibodies, we generated a panel of $c n t n$-expressing HEK cells (Fig. $2 a-f$ ). Cntn cDNAs were inserted into a unique NotI site of pCMV-Venus-t2A-NotI vector. Venus is a bright yellow fluorescent protein (Nagai et al., 2002). The triple-2A (t2A) sequence comprises three tandem copies of a $2 \mathrm{~A}$ self-cleavable peptide sequence (DLLKLAGDVESNPGP) from a foot and mouth disease virus (Ryan and Drew, 1994). These constructs were transfected to HEK293T cells and stained as described previously (Yamagata and Sanes, 2010). As shown in Figure 2, antibodies to Cntns1-5 were monospecific. Overexpressed Cntns are expressed on the cell surface (data not shown), indicating successful cleavage of the polyprotein at the $2 \mathrm{~A}$ sequence.

Anti-mouse Sdk1 antibodies, which cross-react with chick Sdk1, were generated by immunizing Sdk1-null mice (M. Yamagata and J. R. Sanes, unpublished observations) with mouse Sdk1-transfected L cells as described above. Mouse monoclonal antibodies to Sdk2 (CS22) and GFP (GFP-G1) were described previously (Yamagata et al., 2002; Yamagata and Sanes, 2012). Rabbit anti-choline acetyltransferase antibodies were from Miles Epstein (University of Wisconsin, Madison, WI). Mouse 


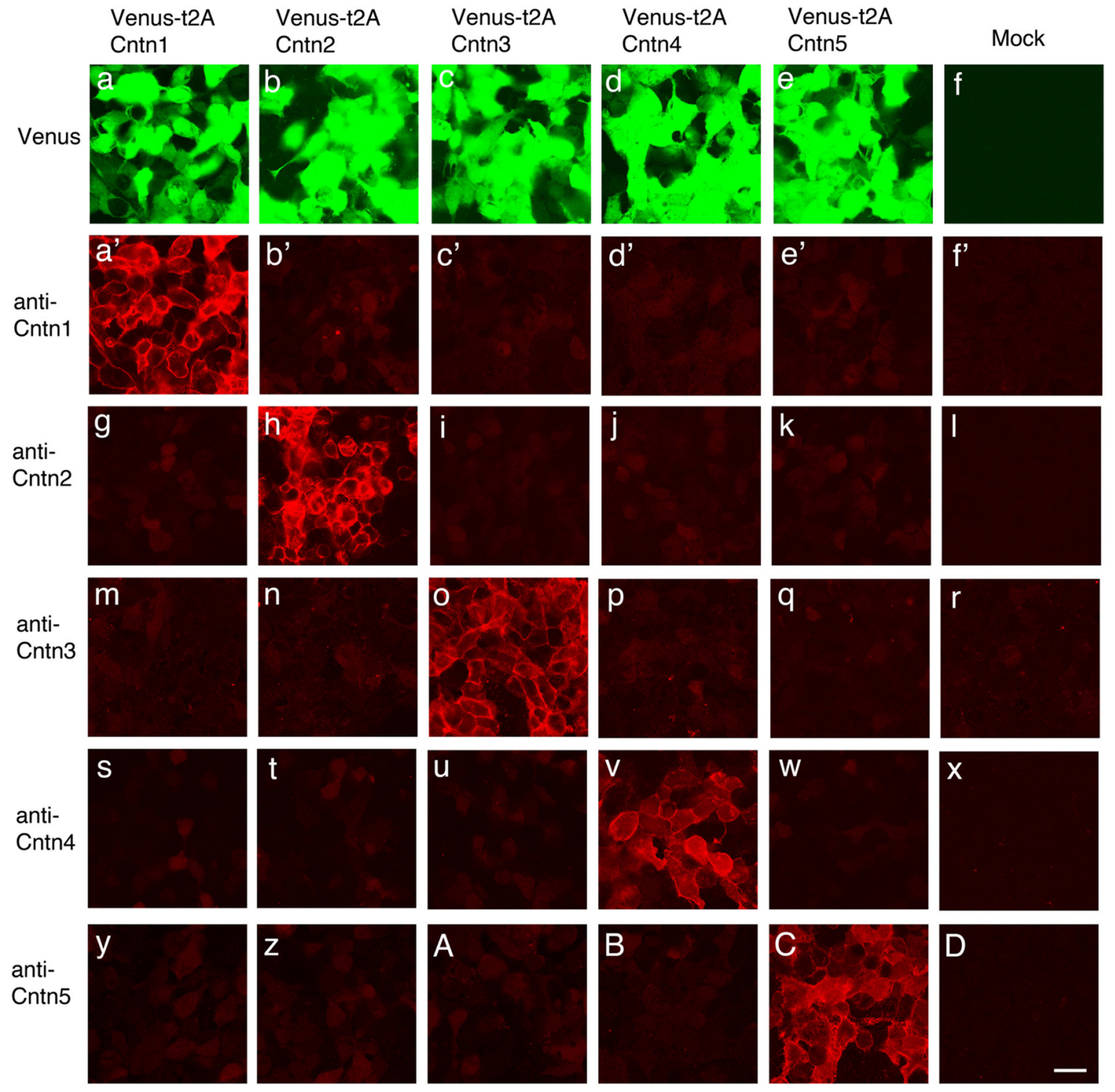

Figure 2. Specificity of Contactin antibodies. Antibodies to Cntns1-5 were tested in HEK cells transfected with cDNAs encoding a (ntn plus a yellow fluorescent protein, Venus (a-f). Cells were then fixed, permeabilized, and stained with anti-Cntn antibodies $\left(\boldsymbol{a}^{\prime}-\boldsymbol{D}\right)$. Each anti-Cntn antibody recognized its cognateCntn but did not cross-react detectably with other (ntns. $\boldsymbol{a}^{\prime} \boldsymbol{-} \boldsymbol{f}$ show samefields as $\boldsymbol{a}-\boldsymbol{f}$.Scale bar, (in $\left.\boldsymbol{D}\right) 10 \mu \mathrm{m}$.

anti-synaptotagmin antibody 48 was from Developmental Studies Hybridoma Bank. Rabbit anti-green fluorescent protein was from Millipore.

Immunohistochemistry. Retina was fixed, sectioned, and immunostained as described previously (Yamagata and Sanes, 2010). For double staining with two different mouse antibodies, Zenon Horseradish Peroxidase Mouse IgG1 Labeling Kit (Life Technologies) was used to label mouse IgG and detected using TSA Plus.

In ovo electroporation. Venus-t2A-cntn sequences (see above) were amplified using Phusion DNA polymerase, cloned into pCR8-TOPO, and transferred to a piggyBac transposon plasmid, pXL-BacII-CAGRfA (Yamagata and Sanes, 2010). The Venus-t2A-cntn plasmid was mixed 10:1 with pCAG-mPBorf, a CAG-driven piggyBac transposase plasmid with a mouse-codon-optimized piggyBac transposase DNA sequence. Optic vesicles of E2 (Hamburger/Hamilton Stage 10) chicks were electroporated as described previously (Yamagata and Sanes, 2008, 2010). Retinas were dissected out at E16 and fixed with $4 \%$ paraformaldehyde/PBS.

To attenuate $c^{n}$ tn 2 expression, we used RNA interference. Six sequences were tested by inserting them into the pcDNA6.2-GW/EmGFPmiR vector (Life Technologies) as described previously (Yamagata and Sanes, 2008, 2010), coexpressing them with cntn2 cDNA in HEK 293T cells, and staining with anti-Cntn2. Sequences Cntn2A and Cntn2C effectively decreased Cntn2 levels as illustrated for $\mathrm{Cntn} 2 \mathrm{C}$ in Figure 3. For quantitative analysis of the decrease, we measured fluorescence intensity of Cntn2 in micrographs such as those shown in Figure 3. This analysis indicated that the Cntn2C miRNA decreased Cntn2 levels to $12 \pm 5 \%$ of control levels (mean \pm SEM, $n=12$ ).

Cntn2A:TGCTGCAATGTAGAGGTTTCCTGTGGGTTTTGGCCA CTGACTGACCCACAGGACCTCTACATTG; Cntn2C:TGCTGAGC AGTAATAGTCACCCTGCTGTTTTGGCCACTGACTGACAGCAGG GTCTATTACTGCT. These sequences and EmGFP were transferred to pXL-BacII-CAG-RfA and embryos were coelectroporated with a piggyBac transposase plasmid as described above.

Experiments were performed in accordance with NIH guideline protocols approved by the Harvard University Standing Committee on the Use of Animals in Research and Teaching.

Cell adhesion assay. Human erythroleukemic K562 cells (ATCC) were transfected using DMRIE-C with the pCAG-cntn-IRES-neo constructs described above. Stable transfectants were selected using G418, and single cells were cloned by limiting dilution. Cells were labeled with Cell Tracker Green CMFDA (5-chloromethylfluorescein diacetate) (Life Technologies) or Cyto-ID Red Long-Term Cell Tracer (Enzo Life Sciences), and adhesion was assayed as described previously for Sdks and 

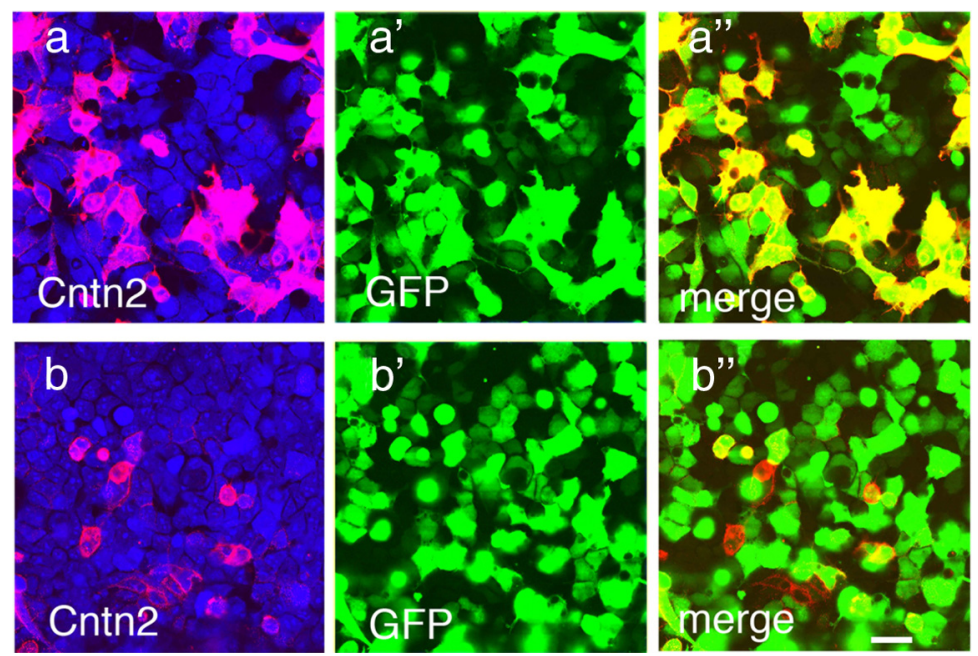

Figure 3. Depletion of contactin2 by RNAi. HEK cells were cotransfected with two plasmids, one encoding Cntn2 and the other encoding GFP $\left(\boldsymbol{a}, \boldsymbol{a}^{\prime}, \boldsymbol{a}^{\prime \prime}\right)$ or GFP and microRNA $\operatorname{Cntn2C}\left(\boldsymbol{b}, \boldsymbol{b}^{\prime}, \boldsymbol{b}^{\prime \prime}\right)$. The microRNA dramatically decreased (ntn2 levels. Note that most of the Cntn2-positive cells in $\boldsymbol{b}$ do not coexpress microRNA, likely due to imperfect cotransfection. Scale bar, $10 \mu \mathrm{m}$.
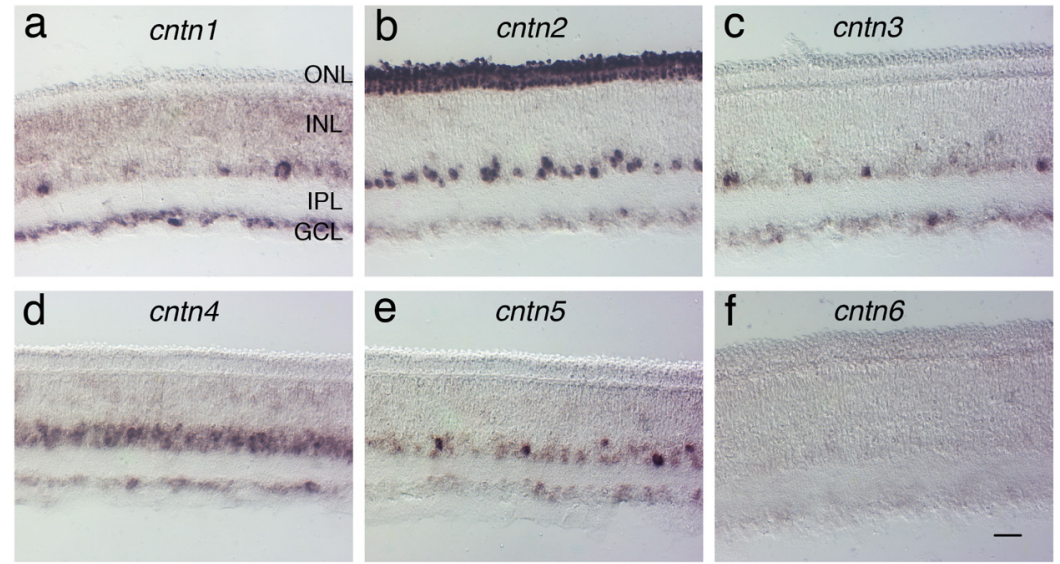

Figure 4. Expression of contactin1- 6 in chick retina. Expression of cntns 1- 6 was assessed by in situ hybridization to sections of E15 chick retina. Cntns 1-5 are expressed by subsets of cells in the inner side of INL and in the GCL (a-e). Based on their position, labeled cells in the INL are amacrine cells; GCL cells may be amacrine and/or retinal ganglion cells. Cntn2 is also expressed by photoreceptors in 0NL. Cntn6 is not detectably expressed in chick retina at this stage $(\boldsymbol{f})$. Scale bar, $25 \mu \mathrm{m}$.

Dscams using HEK 293T cells (Yamagata and Sanes, 2008), except that trypsin digestion was omitted because K562 cells are nonadherent.

\section{Results}

Subsets of retinal neurons express contactins $1-5$

Approximately $60 \%$ of the neurons in chick retina express one of four homologous IgSF genes: Sdk1, Sdk2, Dscam and DscamL (Yamagata et al., 2002; Yamagata and Sanes, 2008). To begin this study, we examined expression of closely related genes. Analysis of the mouse and chick genomes showed that two small groups of IgSF genes are the closest relatives of the Sdks and Dscams: four NgCAM/L1-like genes and six cntn genes (Fig. 1a). Previous studies showed that $N g C A M / L 1$ and $N r C A M$ are broadly expressed in retina but that cntn2 is expressed by a discrete subset of retinal neurons (Drenhaus et al., 2003) (M. Yamagata, unpublished observations). We therefore focused on the cntns. Each contains six Ig domains, four fibronectin type III repeats, and a glycosylphosphatidyl inositol (GPI) anchor that links them to the cell surface, and all are closely related to the Sdks and Dscams (Fig. 1b) (Shimoda and Watanabe, 2009; Stoeckli, 2010).

In situ hybridization revealed that $c n t n s 1-5$ are expressed by neuronal subsets in chick retina at E15 (Fig. 4). All five genes are expressed by cells in the inner half of the inner nuclear layer (INL) and in the ganglion cell layer (GCL). Based on their position, the cells in the INL are identifiable as amacrine cells; the cells in the GCL could be amacrine and/or retinal ganglion cells (Millar et al., 1987). For cntns2-5, more cells were labeled in the INL than in the GCL. Cntn2 was also expressed in the outer nuclear layer, which contains photoreceptors. Cntn6 was not detectably expressed in E15 retina. No cntns were detectably expressed in the outer part of the INL, which contains bipolar, horizontal, and Müller glial cells.

\section{Contactins are localized to sublaminae within the IPL}

To localize Cntn proteins, we obtained or generated antibodies to Cntns1-5, ensured that they were monospecific (Fig. 2 ), and used them to stain sections of retina. Each Cntn was concentrated in two or more discrete bands within the IPL (Fig. $5 a, c, e, g, i)$. Some somata in the INL were also Cntn positive.

At least 10 sublaminae can be recognized in the IPL; we follow Ramon y Cajal (1892) in grouping them into five sets, S1-5 (Fig. $5 k$ ). The outer portions of S2 and S4 contain processes of starburst amacrine cells, the sole cholinergic neurons of retina (Millar et al., 1987). Therefore, double-staining with antibodies to Cntns and to the cholinergic marker, choline acetyltransferase (ChAT), provides a useful way to determine the sublaminae in which Cntn-positive processes reside. Cntn1 and 2 were each concentrated in pairs of sublaminae, with Cntn1-positive bands in $\mathrm{S} 1$ and S5, and Cntn2-positive bands in inner S2 and S4 (Fig. 5b,d). Cntn-3 was concentrated in bands that spanned the region from the lower portion of $\mathrm{S} 2$ to the upper portion of S4 (Fig. 5f). Cntns4 and 5 were each concentrated in three closely spaced groups of bands (Figs. $5 h, j$ ). Remarkably, the sublaminae positive for each of these 5 Cntns formed nearly mirror-symmetric patterns within the IPL. This pattern raises the possibility that Cntns are expressed by "paramorphic pairs" of retinal cell types, that are similar in structure and function but differ in that one arborizes in the ON and the other in the OFF sublaminae (Famiglietti, 2005).

\section{Contactins are differentially expressed by non-overlapping neuronal subsets}

We next asked whether cntns are expressed by overlapping or distinct subsets of retinal neurons. In some cases, mutually exclusive expression of two cntns was suggested by the distinct sublaminae within which their proteins were localized (for example, Cntns1/2, 2/4, and 2/5; Fig. $6 a-c)$. Other pairs, however, were partially colocalized within sublaminae (for example, Cntns4 and 5; Fig. $6 d$ ). In these cases, the cntns might either be coexpressed, 

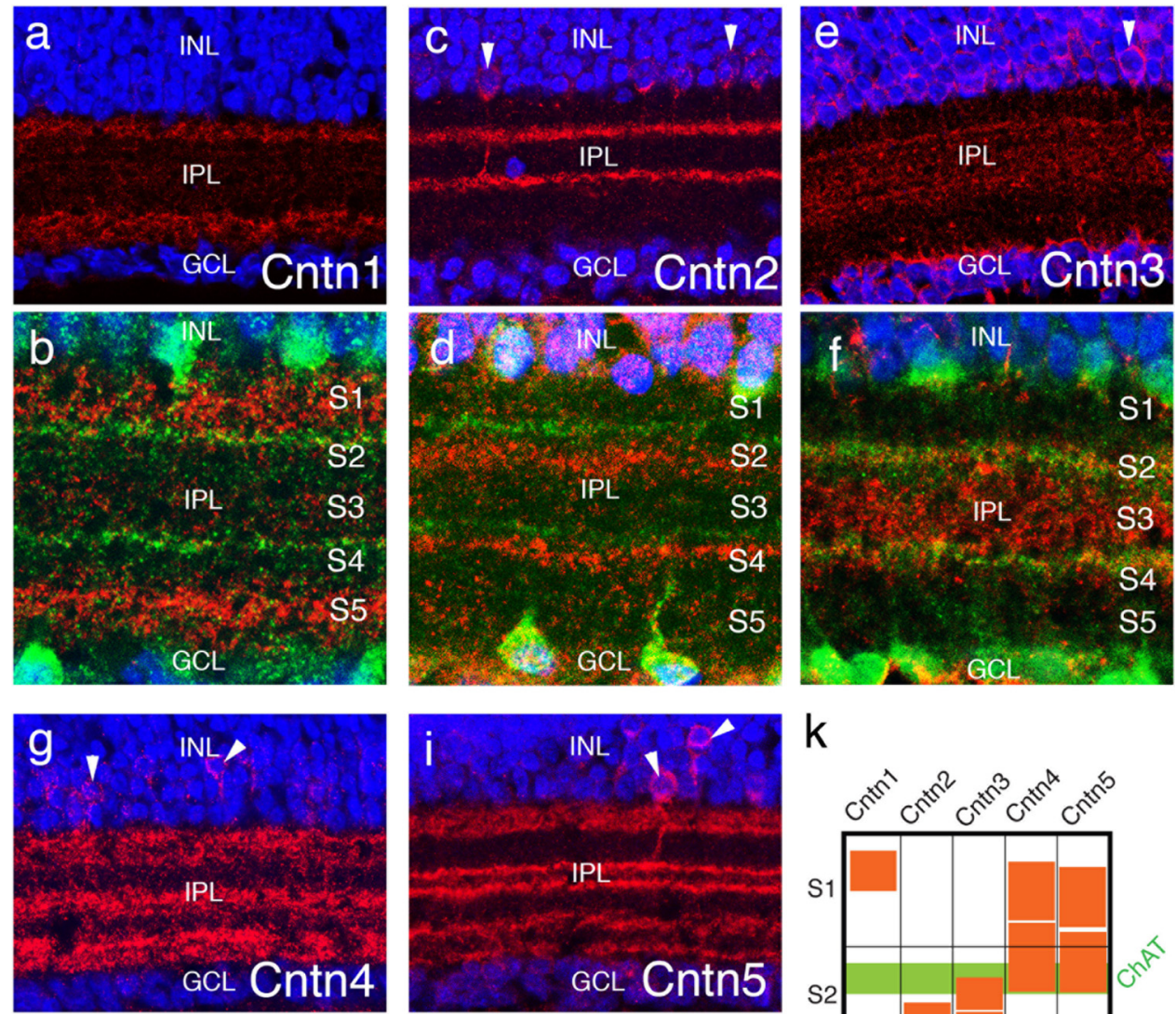

\section{$\mathrm{k}$}
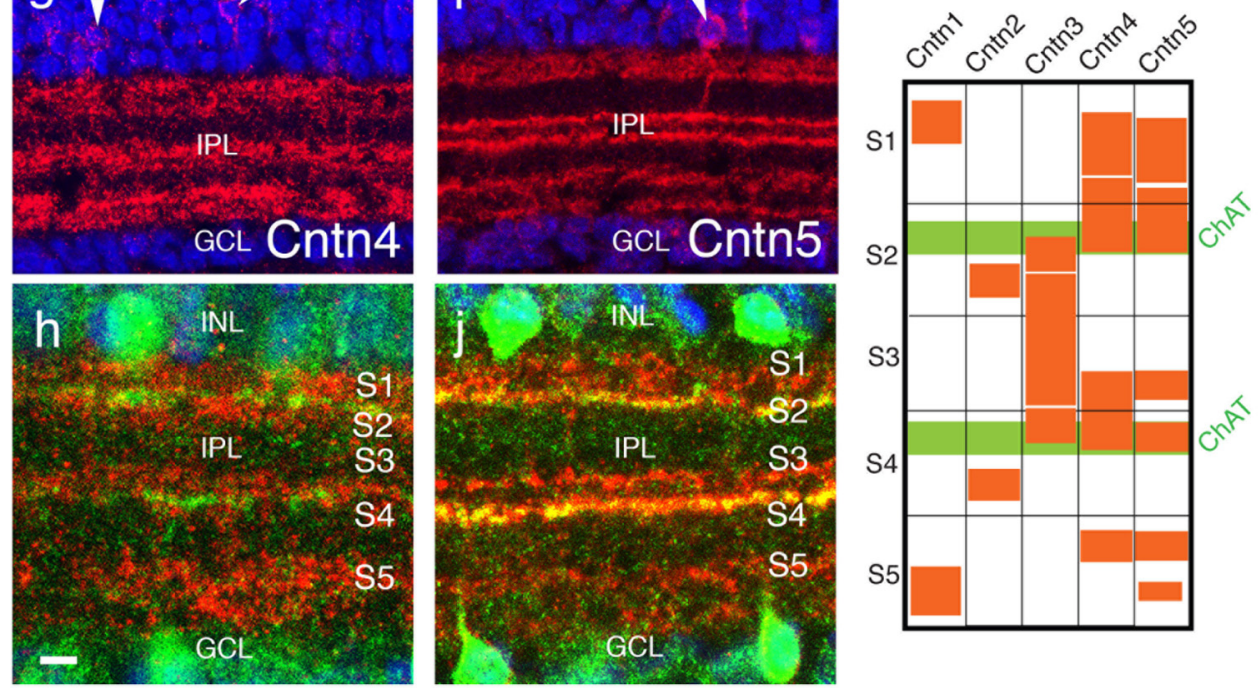

Figure 5. Contactins are concentrated in discrete sublaminae within the inner plexiform layer. Sections of E16 retina were stained with antibodies to Cntns-1-5. $\boldsymbol{a}, \boldsymbol{c}, \boldsymbol{e}, \boldsymbol{g}, \boldsymbol{i}$, Each antibody stains distinct sublaminae in the IPL, as well as somata of presumptive amacrine cells in the INL. Layers were identified by counter-staining with a fluorescent Nissl dye (blue). The antibodies also lightly stain some cell bodies in INL (arrowheads). $\boldsymbol{b}, \boldsymbol{d}, \boldsymbol{f}, \boldsymbol{h}, \boldsymbol{j}$, Higher-power views of IPL from similar sections double-stained with anti-Cntn plus anti-ChAT to facilitate identification of sublaminae. $\boldsymbol{k}$, Schematic summarizing sublaminae localization of Cntns-1-5 in the IPL. Abbreviations as in Figure 4 . Scale bar: $10 \mu \mathrm{m}$ for $\boldsymbol{a}, \boldsymbol{c}, \boldsymbol{e}, \boldsymbol{g}$, and $\boldsymbol{i} ; 5 \mu \mathrm{m}$ for $\boldsymbol{b}, \boldsymbol{d}, \boldsymbol{f}, \boldsymbol{h}$, and $\boldsymbol{j}$.

or expressed by distinct neurons with processes that stratify in close proximity. To distinguish these possibilities, we used two color in situ hybridization. Examples are shown in Figures $6 e-n$ and results are summarized in Figure 6o. In most cases, cntns were expressed at high levels by non-overlapping subsets of retinal neurons; the upper limit to expression was $\sim 5 \%$, some of which might reflect inability to distinguish adjacent cells. (Additional cells were lightly labeled by $\mathrm{cntn}$ probes, but we could not distinguish this expression from background and therefore did not score it.) One exception was the $c n t n 4 / c n t n 5$ pair; $\sim 10 \%$ of the neurons that expressed either one of these cntns coexpressed the other (Fig. 6n). Thus, cntns are expressed largely but not entirely by mutually exclusive neuronal subsets in retina.

\section{Expression and localization of Contactin2}

For detailed analysis of contactin function, we focused on Cntn2. To provide a foundation for these studies, we first compared the distri- bution of Cntn2 to those of Sdk1, Sdk2, Dscam, and DscamL. Immunostaining indicated that Cntn2 was concentrated in IPL sublaminae that were poor in Dscam and DscamL but rich in Sdks1 and 2 (Fig. 7a,b) (data not shown). Thus, cntn2 is unlikely to be coexpressed with $d s c a m$ or $d s c a m L$ but could be coexpressed with $s d k 1$ or $s d k 2$. In situ hybridization confirmed that $c n t n 2$ is expressed entirely by $d s c a m$ - and $d s c a m L$-negative neurons, and also showed that all cntn2-positve neurons are $s d k 2$ negative (Fig. $7 d-f$ ). Thus, cntn2, sdk2, Dscam, and DscamL define four mutually exclusive neuronal subsets, even though processes of some Cntn2- and Sdk2positive neurons arborize in the same sublaminae. In contrast, significant overlap was observed between cntn2- and sdk1expressing neurons in the inner part of the INL, where amacrine cell somata reside (Fig. $7 c$ ). In this region, approximately half of the cntn2-positive cells were also $s d k 1$ positive and approximately half of the sdk1-positive cells were $\mathrm{cntn} 2$ positive.

We next asked when Cntn2 immunoreactivity appears in the 

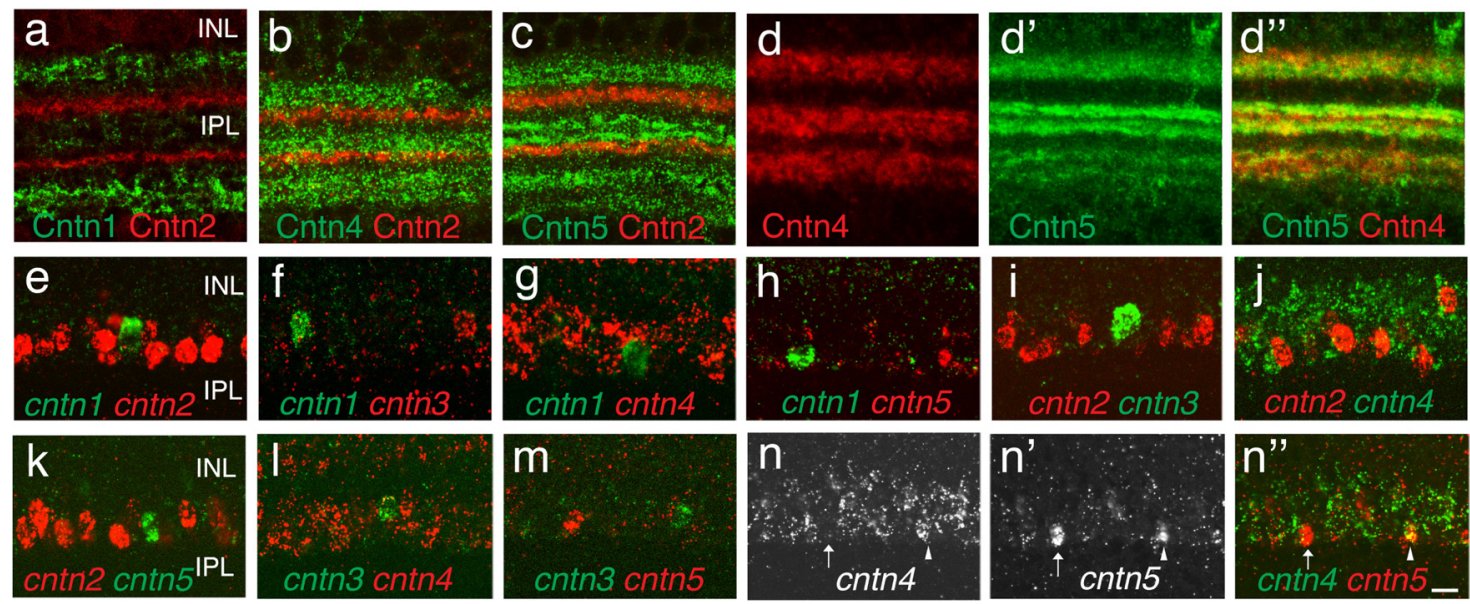

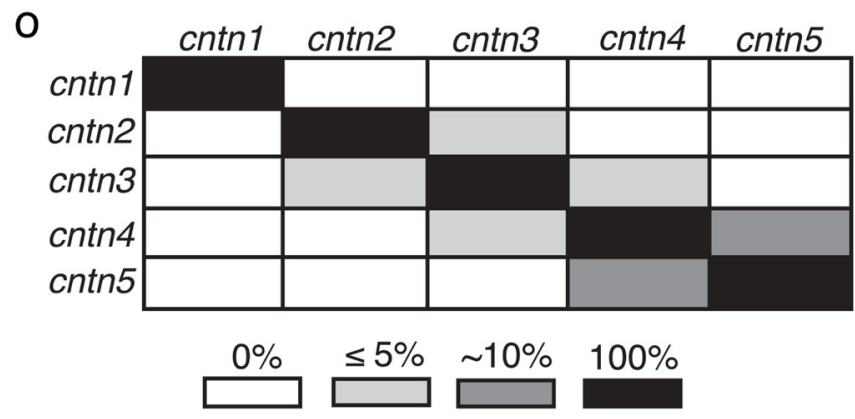

Figure 6. Contactins are expressed by largely non-overlapping subsets of retinal neurons. $\boldsymbol{a}-\boldsymbol{d}$, Sections of E16 retina were stained with pairs of antibodies to $C$ ntns. Cntn 1 and $(n+n 2$ (a), Cntn2 and Cntn4 (b), and Cntn2 and (ntn5 (c) are concentrated in non-overlapping sublaminae in the IPL. In contrast, (ntn4 and (ntn5 are concentrated in partially overlapping sublaminae (d). $\boldsymbol{e}-\boldsymbol{n}$, E16 retinal sections were incubated with pairs of $c$ ntn probes, conjugated to distinct haptens for two-color in situ hybridization. Arrows and arrowheads mark corresponding points in $\boldsymbol{n}, \boldsymbol{n}^{\prime}, \boldsymbol{n}^{\prime \prime}$. $\mathbf{0}$, Summary of quantitative results from sections such as those in $\boldsymbol{e}-\boldsymbol{n}(\boldsymbol{n}=20-50$ heavily labeled cells per pair). The only substantial coexpression of $c n t n s$ is a $\sim 10 \%$ overlap between cntn 4 and cntn5. Scale bar: $10 \mu \mathrm{m}$ for $\boldsymbol{a}-\boldsymbol{d} ; 6 \mu \mathrm{m}$ for $\boldsymbol{e}-\boldsymbol{n}$.

IPL, and whether it is localized to specific sublaminae from the time it first appears. Little Cntn2 was detectable in the IPL of central retina at E9 (Fig. 8a). Levels increased gradually from E11 to E15, then declined over the next several days (Fig. 8b-e). Throughout this period, immunoreactivity was localized to two narrow bands, as shown above for E15 retina. A previous paper reported a third band of Cntn2 immunoreactivity in the chick IPL (Drenhaus et al., 2003); we observed a similar pattern with a polyclonal antibody to Cntn2 (data not shown) but not with the monoclonal antibody shown here. It is therefore possible that the third band represents a Cntn2-related epitope on another protein, or that a distinct isoform of Cntn2 is present on processes in this band. Cntn2 was also present in photoreceptors and in the outer plexiform layer (OPL) between E11 and E15, with the peak expression in the OPL being a few days earlier than that in the IPL (Fig. 8a-e).

Examination of single confocal images revealed a difference between the subcellular localization of Cntn2 and Sdks. In the outer retina, $\mathrm{Sdk} 2$ is concentrated at synapses formed by photoreceptor terminals on processes of horizontal and bipolar cells in the OPL (Yamagata and Sanes, 2010) (Fig. 8i). In contrast, Cntn2 was present on lateral surfaces of photoreceptor processes, but not concentrated at synaptic sites (Fig. 8h). Similarly, in the IPL, Cntn2 was diffusely distributed along processes in the IPL (Fig. $8 f$ ), whereas Sdk2 was concentrated in puncta (Fig. $8 g$ ). In both OPL and IPL, Sdk2 was more closely associated with nerve terminals (marked with antibodies to the synaptic vesicle protein, synaptotagmin) than was Cntn2 (Fig. $8 f-i$ ) (data not shown). These differences are consistent with the idea that Cntn2 is pres- ent along neuritic surfaces, whereas Sdk2 is concentrated at synaptic sites.

\section{Contactin2 is required for laminar targeting of neurites}

We used RNA interference (RNAi) to determine whether Cntn2 is necessary for correct targeting of neurites to appropriate sublaminae. We tested six miRNA sequences for their ability to decrease Cntn2 levels in heterologous cells, and found two, Cntn2A and Cntn2C, that were effective (Fig. 3). We then used a piggyBac transposon system to introduce Cntn2C miRNA together with GFP into retinal progenitors at E2. At E15, following development of sublaminae, we stained retinas for Cntn2 and Sdk1, which as noted above, is expressed by a large subset of Cntn2positive neurons. Whereas Sdk1 is highly concentrated in S4 of control retinas, it is more diffusely distributed in Cntn2-depleted retinas (Fig. 9). Our interpretation is that when Cntn2 levels fall below some critical level, neurites can still arborize in the IPL, but the arbors are no longer tightly confined to specific sublaminae.

Three additional observations confirmed the specificity of this effect. First, similar results were obtained with Cntn2A, a second miRNA directed at cntn2 (data not shown). Second, the localization of Sdk1 was unaffected by introduction of control miRNAs (compare Figs. $7 a, 9 c$ ). Third, the localization of Sdk2, which is expressed exclusively by Cntn2-negative neurons, was unaffected by depletion of Cntn2 (Fig. 9d,h). Together, these results demonstrated that Cntn2 is necessary for targeting arbors of Cntn2positve neurons to appropriate sublaminae. 


\section{Overexpression of contactin2 redirects neurites}

We used a gain-of-function strategy to determine whether Cntn2 is not only necessary but also sufficient for sublaminar targeting of neurites in the IPL. To this end, we introduced Cntn2, together with a fluorescent protein (Venus), into neurons; retinas were transfected at E2 and examined at E15-E16. Whereas we transfected large patches of retinal cells with miRNAs for loss-of-function analysis (Fig. 9), we transfected small groups of neurons for the gain-of-function experiment, so that transgenic neurons would have ample opportunity to interact with wild-type neighbors. As expected, introduction of Venus alone labeled neurons that, in the aggregate, sent processes to all IPL sublaminae (Fig. 10a). Thus, most transduced neurons are unlikely to have expressed endogenous cntn2. In contrast, neurons transduced with Cntn2 and GFP sent a disproportionate fraction of their neurites to S2 and S4, the sublaminae rich in endogenous Cntn2 (Fig. 10b). Double staining with anti-choline acetyltransferase (ChAT) showed that the GFP-positive neurites, like those rich in endogenous Cntn2, were localized directly beneath the ChATpositive band (compare Figs. $5 d, 10 c$ ). Thus, adding Cntn2 to a neuron's adhesive repertoire is sufficient to redirect its processes to sublaminae rich in Cntn2.

Does Cntn2 play a specific and instructive role or could any Cntn direct neurites to the Cntn2-rich sublaminae? To distinguish between these alternatives we introduced other Cntns into retinal neurons. We chose Cntn 1 and 3 for this experiment because processes rich in these Cntns are localized to narrower bands in the IPL than those rich in Cntns4 or 5. Ectopic expression of $c n t n 1$ or 3 redirected processes to sublaminae that expressed endogenous Cntn1 or Cntn3, respectively (compare Figs. 5a,c, 10d,e). Thus, Cntns play an instructive role in localizing neurites to IPL sublaminae.

\section{Adhesive specificity of Contactins}

The diversion of neurites that overexpress cntns1-3 to sublaminae rich in the corresponding endogenous Cntns is consistent with the idea that Cntns act by a mechanism that involves selective homotypic recognition. Indeed, Cntn2 has been shown to bind homophilically (Rader et al., 1993; Felsenfeld et al., 1994), but it is not known whether it also binds heterophilically to other Cntns. To address this issue, we introduced Cntn2 into K562 cells, which do not express known adhesive molecules such as cadherins or integrins (Schreiner and Weiner, 2010). As expected, untransfected cells failed to aggregate when mixed, whereas introduction of Cntn2 led to dramatic aggregation (Fig. $11 a, b)$. Sdk1, Sdk2, Dscam, and DscamL also promoted aggre- gation in this system, as shown previously in other cell lines (Yamagata and Sanes, 2008); Cntn4 also promoted aggregation, whereas cells expressing Cntn 1, 3, or 5 did not aggregate detectably under these conditions, even though immunostaining confirmed that they reached the cell surface (data not shown).

To test heterophilic interactions, we introduced Cntn2 and another IgSF adhesion molecule into separate populations of K562 cells, labeled the two populations with contrasting fluorophores, and mixed them. As expected, red and green Cntn2positive cells formed mixed aggregates, as did red and green Cntn4-positive cells (Fig. $11 c, d, k, l$ ). In contrast, aggregates of cntn2-expressing cells remained largely distinct from aggregates 


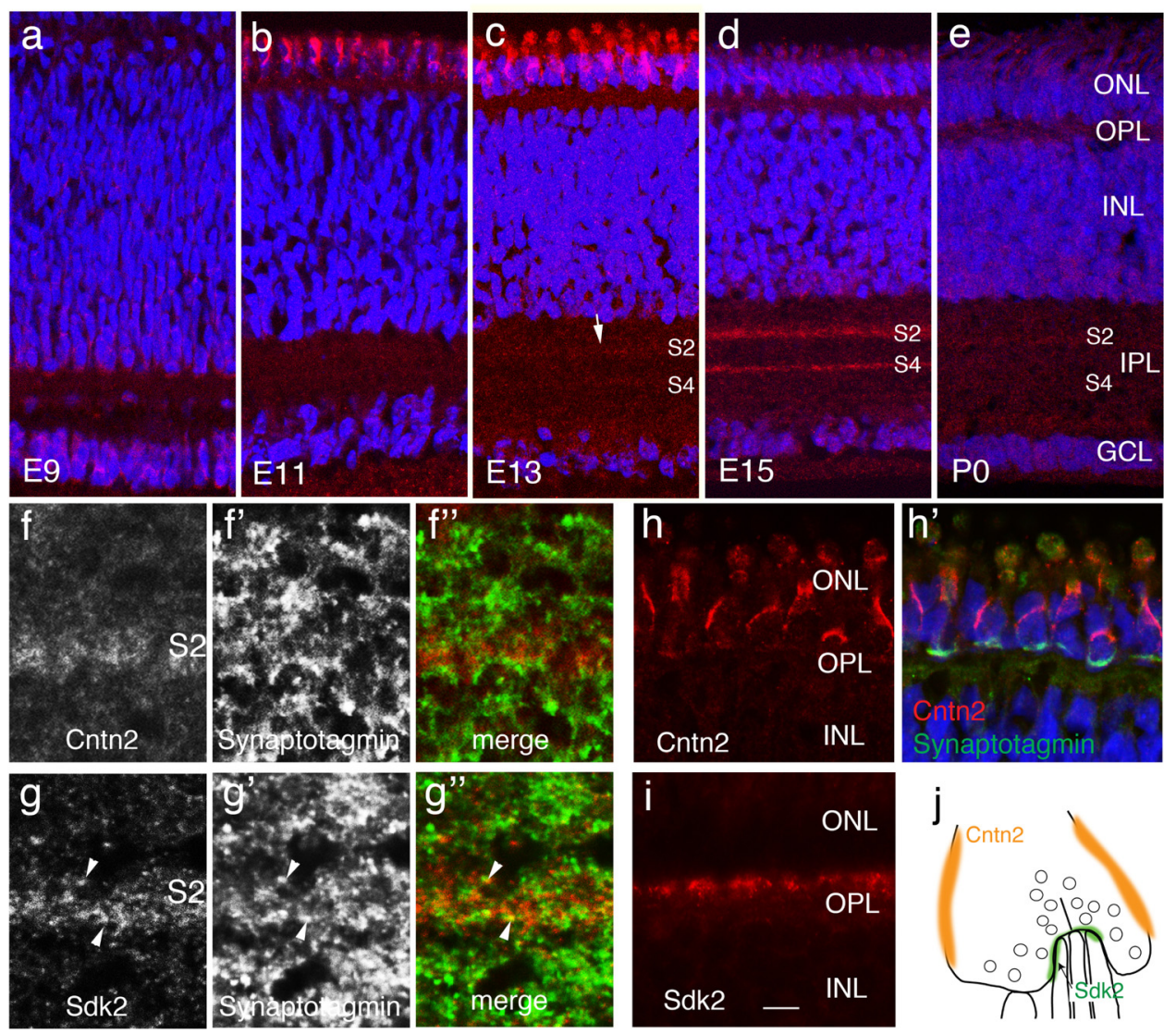

Figure 8. Subcellular localization of Contactin2 in developing retina. $a-e$, Sections of retina from embryos of indicated ages were stained with anti-Cntn2 (red) and fluorescent Nissl dye (blue). Cntn2 levels are low in the IPL at E9 (a); levels increase from E11 to E15 $(\boldsymbol{b}-\boldsymbol{d})$, and then decline by P0 (e). Throughout this period, immunoreactivity is localized to two narrow bands. Cntn2 is also transiently localized to photoreceptors in the ONL and processes in the OPL $(\boldsymbol{b}-\boldsymbol{d})$, with levels declining by E15 $(\boldsymbol{e}) . \boldsymbol{f}, \boldsymbol{g}$, Thin confocal sections of IPL at E15, stained with anti-Cntn2 and anti-Sdk2. Cntn2 is more diffusely distributed in $\mathbf{S} 2$ than $\mathbf{S d k 2}$, which is punctate. Many Sdk2-positive puncta are colabeled with anti-synaptotagmin, which marks nerve terminals (arrowheads). $\boldsymbol{h}$-j, High-power view of photoreceptor cells at E14, stained with anti-Cntn2 (red) plus anti-synaptotagmin (green) to mark nerve terminals ( $\boldsymbol{h}$ ). Cntn2 is localized to lateral surfaces of photoreceptor cells (blue, Nissl staining) but not concentrated at synaptic sites. This pattern contrasts with that of Sdk2, which is concentrated at synapses (i,j) (Yamagata and Sanes, 2010). Abbreviations as in Figure 4. Scale bar: $10 \mu \mathrm{m}$ for $\boldsymbol{a}-\boldsymbol{e} ; 2 \mu \mathrm{m}$ for $\boldsymbol{f}, \boldsymbol{g} ; 5 \mu \mathrm{m}$ for $\boldsymbol{h}, \boldsymbol{i}$.

of cells expressing Sdk1, Sdk2, Dscam, DscamL, or cntn4 (Fig. $11 e, g-j, m)$. Likewise, aggregates of cntn2-expressing cells failed to incorporate cells that expressed $c n t n 1, c n t n 3$, or $c n t n 5$ (Fig. $11 f$ ) (data not shown). These results also show that Cntn2 does not bind strongly to endogenous components of the K562 membrane. Thus, Cntn2 mediates homophilic adhesion but does not interact heterophilically with related IgSF proteins.

\section{Discussion}

In previous studies, we showed that four closely related IgSF members, 2 Sdks and 2 Dscams, are expressed by nonoverlapping subsets of retinal neurons and promote laminaspecific arborization of processes in the IPL (Yamagata et al., 2002; Yamagata and Sanes, 2008, 2010). These results suggested the existence of an IgSF code for laminar specificity in retina, but left open the question of how laminar specificity was regulated in the $\sim 40 \%$ of retinal cells that are Sdk and Dscam negative. Here, we asked whether Cntns, six close relatives of Sdks and Dscams, might be involved. Cntns have been implicated in numerous aspects of neural development, including neurite outgrowth, axonal fasciculation, myelin formation, and development of sensory and motor pathways (Rathjen et al., 1987; Gennarini et al., 1991; Yoshihara et al., 1994, 1995; Ogawa et al., 1996; Berglund et al., 1999; Takeda et al., 2003; Kaneko-Goto et al., 2008; Toyoshima et al., 2009) (for review, see Salzer et al., 2008; Shimoda and Watanabe, 2009; Stoeckli, 2010; Zuko et al., 2011). They had not, however, been studied in the context of laminar specificity. We found that five contactins are expressed in subsets of retinal neurons, and that they, like Sdks and Dscams, promote restriction of neuronal arbors to specific sublaminae within the IPL. Our results expand the IgSF code from 4 to 9 members, and raise the question of whether it may now be complete.

\section{An IgSF code for laminar specificity}

Genomic analysis shows that the contactins are the closest relatives of the Sdks and Dscams (Fig. 1). The Cntns are also remarkably similar to Sdks and Dscams in retinal expression and function. We summarize these similarities here, then go on to discuss some differences among them.

\section{Expression}

$S d k 1, s d k 2, d s c a m, d s c a m l$, and cntns1-5 are all expressed by discrete subsets of retinal cells. In each case, the positive subsets include cells in both the inner nuclear and ganglion cell layers. Moreover, the subsets that express each gene are, to a large extent, non-overlapping (Fig. 12). Cells that express each of the four $s d k$ and dscam genes are almost completely non-overlapping, as are cells that express each of the five cntn genes. The extent to which $s d k / d s c a m$-positive cells express cntns and vice versa is less clear. 

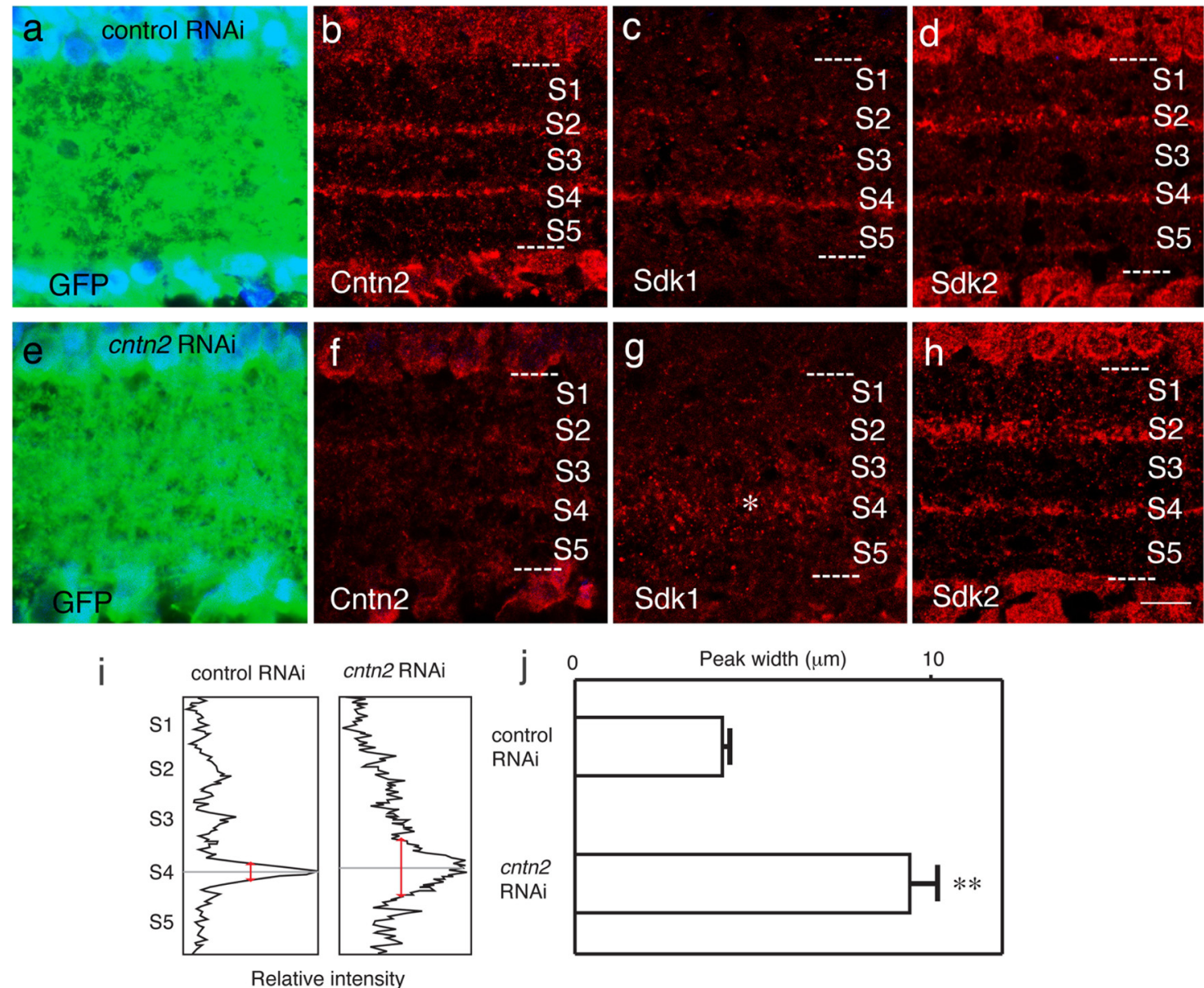

Figure 9. Contactin2 is required for laminar targeting of neurites. GFP was introduced to large patches of retinal neurons, along with either a control miRNA ( $\boldsymbol{a}-\boldsymbol{d})$ or miRNA $(n t n 2 C(\boldsymbol{e}-\boldsymbol{h})$. Sections were stained with antibodies to GFP to identify transduced regions $(\boldsymbol{a}, \boldsymbol{e})$; (ntn2 to assess the efficacy of the miRNA $(\boldsymbol{b}, \boldsymbol{f})$, Sdk1 to monitor processes of (ntn2-expressing neurons $(\boldsymbol{c}, \boldsymbol{g})$, and Sdk2 to assess the specificity of the effect $(\boldsymbol{d}, \boldsymbol{h})$. This strategy is based on the observation that $\sim 50 \%$ of (ntn2-positive neurons are Sdk1 positive but none is $S d k 2$ positive (see Fig. 7). Depletion of Cntn2 decreases the concentration of Sdk1-bearing processes in S4 (asterisk) and leads to their dispersion into neighboring zones. To quantify the dispersal, we measured the density of Sdk2 immunoreactivity as a function of laminar depth (i), then calculated the width of the curve at half-maximal intensity (j). The difference between control $(n=10)$ and $(n t n 2$-depleted samples $(n=$ 21) was significant $(* * p<0.01)$. Scale bar, $10 \mu \mathrm{m}$.

Nearly all cntn2-positive cells are sdk2, dscam, and $d s c a m L$ negative, but around half are $s d k 1$ positive. Based on laminar distribution of the protein products, it is likely that cntn1-, cntn4-, and cntn5-positive cells are $s d k 1$ and $s d k 2$ negative. Thus, despite substantial overlap, the nine IgSF genes analyzed here may define largely distinct neuronal populations.

\section{Localization}

Sdk, Dscam, and Cntn proteins are all concentrated in the IPL. Most strikingly, in each case, discrete sublaminae within the IPL are labeled. This pattern results from the lamina-restricted arborization of dendrites in the IPL, a fundamental property that Ramon y Cajal (1892) recognized and used as a basis for classifying retinal subtypes. Thus, the staining patterns support the idea that IgSF members mark specific retinal subtypes.

\section{Adhesion}

Sdks, Dscams, and Cntns1, 2, and 4 all bind homophilically (Rader et al., 1993; Felsenfeld et al., 1994; Freigang et al., 2000; Yamagata et al., 2002; Hayashi et al., 2005; Yamagata and Sanes, 2008) (Fig. 11). Moreover, to the extent tested to far, heterophilic interactions within this group of IgSF proteins are weak. For example, Sdk1 does not bind appreciably to Sdk2, Dscam,
DscamL, or Cntn2; and Cntn2 does not bind appreciably to Sdks, Dscams, or other Cntns. Thus, these IgSF molecules are well suited to mediate selective interactions among neurites in close proximity.

\section{Role in laminar specificity}

Perhaps, most important, effects of overexpressing or depleting Cntn2 on lamina-restricted arborization are similar to those of overexpressing or depleting Sdks and Dscams (Yamagata and Sanes, 2008). In each case, ectopic expression of a gene in a small subset of retinal neurons redirects the neurites of that cell to the sublamina in which it encounters a high concentration of the endogenous gene product on the neurites of its wild-type neighbors. Conversely, RNAi-mediated knockdown of Sdks, Dscams, or Cntn2 results in decreased confinement of neurites to specific sublaminae. Although loss-of-function studies of Cntns are so far limited to Cntn2, effects of ectopically expressing Cntns1 and 3 (Fig. 10d,e) suggest that the results can be generalized to the entire group.

Together, these results support the idea that IgSF members from the Sdk/Dscam/Cntn subfamily mark retinal neurons with distinct sublaminar preferences and play critical roles in restricting neurites to appropriate IPL sublaminae. 

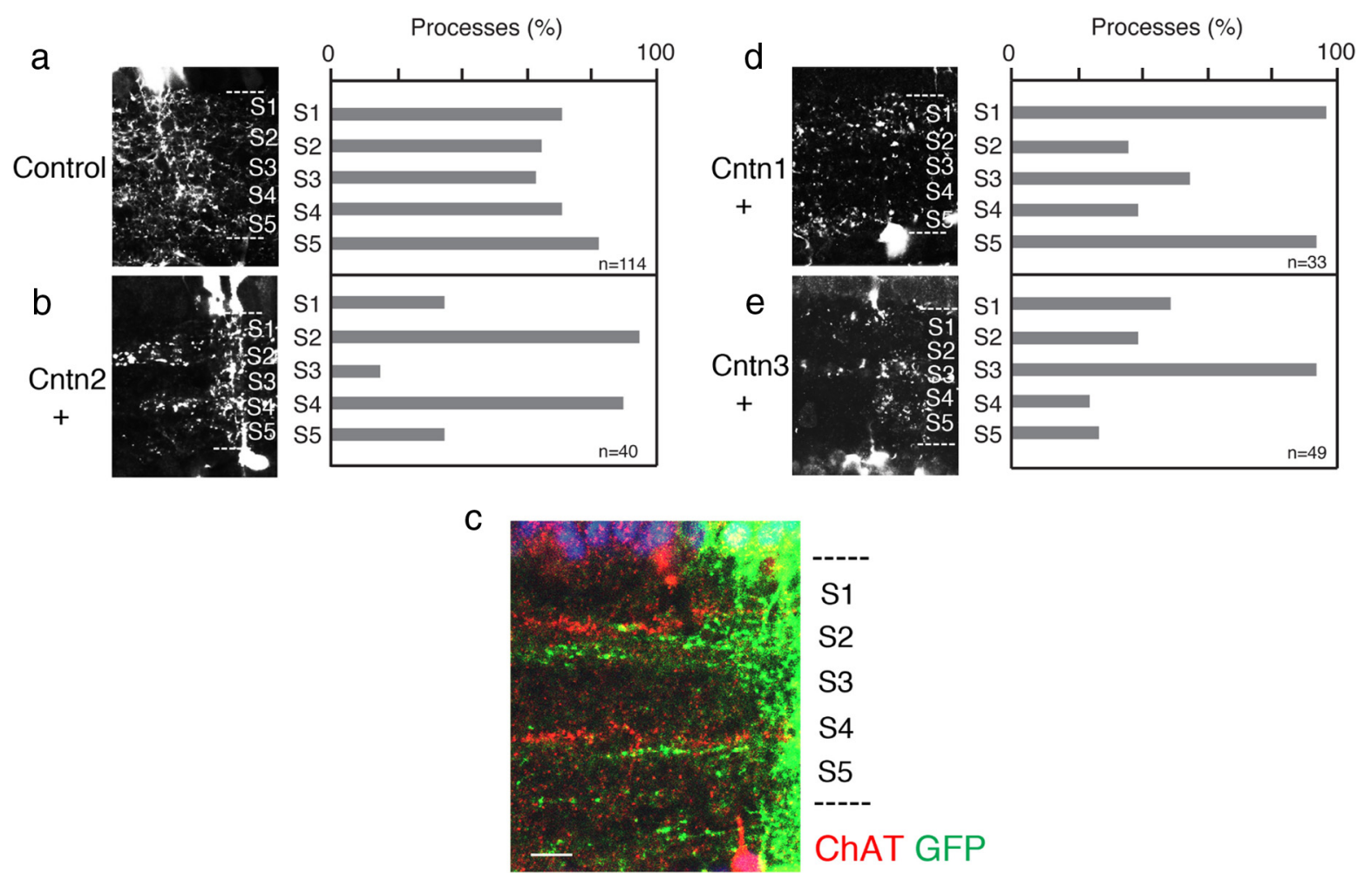

Figure 10. Overexpressed contactin redirects laminar targeting of neurites. $\boldsymbol{a}, \boldsymbol{b}$, Small groups of retinal cells were transduced at $\mathrm{E} 2$ with constructs that encoded Venus alone ( $\boldsymbol{a}$ ) or Venus plus Cntn2. Retinas were analyzed at E17. Left panels show examples, and results are quantified in right panels. Venus-expressing processes were present in all IPL sublaminae at equal frequency, whereas ectopic expression of Cntn2 biased neurites to $\$ 2$ and S4. c, Section from Cntn2-transfected retina stained with anti-ChAT and anti-GFP. The localization of the Venus-positive arbors, directly beneath the ChAT-positive arbors, precisely matches that of endogenous $\mathrm{Cntn2}$ (see Fig. $5 d$ ). $\boldsymbol{d}, \boldsymbol{e}, 0$ verexpression of $\mathrm{Cntns} 1$ and 3 bias neurites to $\mathrm{S}$ and $\mathrm{S} 5$ or $\mathrm{S3}$, respectively. These patterns are similar to those of endogenous (ntns 1 and 3 in the IPL (see Fig. 5a, c). Concentration of processes in S1/S5, S2/S4, and S3 was significant for cntn1, cntn2, and cntn3, respectively ( $p<0.01$ by $\chi^{2}$ test) but not for control. Scale bar: $10 \mu \mathrm{m}$ for $a ; 5 \mu \mathrm{m}$ for $c$

\section{Mechanisms of laminar specificity}

How do Sdks, Dscams, and Cntns mediate laminar specificity? We have proposed that homophilic interactions between Sdks and Dscams on presynaptic and postsynaptic partners mediate synaptic interactions with specific sublaminae of the IPL (Yamagata et al., 2002; Yamagata and Sanes, 2008, 2010). The parallels listed so far encourage the speculation that contactins act in similar ways. However, several observations suggest that Sdks/Dscams and Cntns may promote laminar restrictions by somewhat different mechanisms.

\section{Protein-protein interactions}

To date, no heterophilic transmembrane ligands have been identified for Sdks or Dscams, although Dscam has been reported to act as a netrin receptor in both invertebrates and vertebrates (Andrews et al., 2008; Ly et al., 2008; Liu et al., 2009). In contrast, numerous heterophilic ligands have been documented for the contactins, including NgCAM, protein tyrosine phosphatases, and amyloid precursor proteins (Kuhn et al., 1991; Suter et al., 1995; Kunz et al., 1998; Pavlou et al., 2002; Osterfield et al., 2008; Shimoda and Watanabe, 2009; Zuko et al., 2011). Some of these are present in the IPL (Drenhaus et al., 2003). Moreover, in some cases where homophilic interactions of Cntn2 have been shown to be required for intercellular adhesion, they appear to be cis-interactions (between Cntn2 molecules in the same cell) rather than in trans-interactions (between Cntn2 molecules on apposing membranes) (Buttiglione et al., 1998). Thus, contactins may promote laminar specificity in part via interactions with heterophilic ligands.

Another distinction between Sdks/Dscams and Cntns is that the former are transmembrane proteins whereas Cntns are GPI- linked. Thus, whereas Sdks and Dscams can bind directly in cytoplasmic scaffolding and signaling proteins, Cntns must signal indirectly. Key players in this process are the six members of the contactin-associated protein (Caspr or Cntnap) family, transmembrane proteins that interact selectively in cis with Cntns (Peles et al., 1997; Poliak et al., 1999; Spiegel et al., 2002; Traut et al., 2006). Like Sdks and Dscams (Yamagata and Sanes, 2010), Casprs bind to scaffolding proteins of the PDZ family via their C-termini (Spiegel et al., 2002). In addition, however, the Casprs bind heterophilic ligands extracellularly, adding further complexity to the range of intercellular interactions that could mediate effects of Cntns on laminar specificity.

\section{Subcellular localization}

Although Sdks, Dscams, and Cntns are all present at highest levels in the synaptic layers of the retina, their fine localization differs. Sdks are highly concentrated at synaptic sites, whereas Cntns are more evenly distributed along neuronal processes. The difference is perhaps best seen at photoreceptor synapses, whose large size facilitates analysis at the light-microscopic level (Fig. 8). Previous ultrastructural studies have also shown a broad distribution of Cntn2 along axonal processes of sensory neurons in the chick hindlimb (Xue and Honig, 1999).

\section{Expression by retinal ganglion cells}

Each $s d k$ and $d s c a m$ is expressed by $10-15 \%$ of retinal ganglion cells as well as a similar fraction of interneurons. It is thus reasonable to imagine that these proteins mediate transsynaptic interactions. In contrast, while cntns $1-5$ are all expressed by sizeable populations of amacrine cells, only $c_{n} n 1$ is expressed by a large 

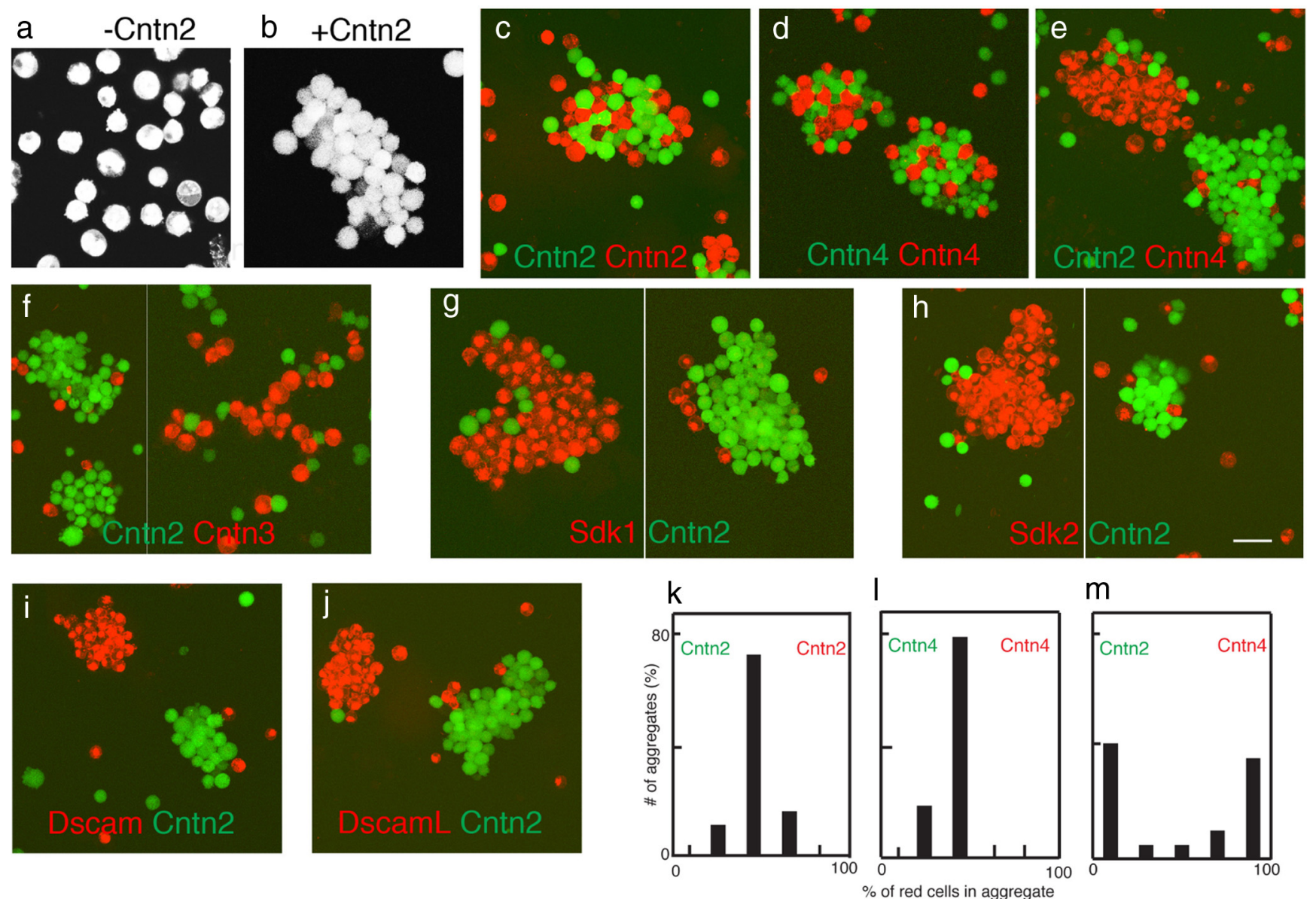

Figure 11. Cntn-2 mediates homophilic but not heterophilic adhesion. $\boldsymbol{a}, \boldsymbol{b}, \mathrm{K} 562$ cells stably expressing cntn2 were fluorescently labeled, dissociated mechanically, and agitated gently for $1 \mathrm{~h}$. Nontransfected cell remained isolated, but Cntn2-expressing cells aggregated. c-j, Stably transfected K562 cells were dissociated mechanically and stained, then two populations stained with contrasting fluorophores and agitated for $1 \mathrm{~h}$ to promote aggregation. Cntn-2, Cntn-4, Sdk-1, Sdk-2, Dscam, and DscamL promoted homophilic aggregation, while Cntn-3-expressing cells aggregated poorly under these conditions. Cells expressing Cntn2 did not aggregate detectably with cells expressing any of the other IgSF genes tested. Thus, cntn2 promotes homophilic but not heterophilic adhesion. $\boldsymbol{f}-\boldsymbol{h}$ are composites, showing two clusters that were from the same well but were not adjacent. $\boldsymbol{k}-\boldsymbol{m}$, Histograms show percentage of red cells in aggregates from Cntn2-green/Cntn2-red ( $\boldsymbol{k})$, Cntn4-green/Cntn4-red (I), or Cntn-2-green/Cntn4-red ( $\boldsymbol{m})$ combinations. These results confirm that $\mathrm{Cntn2}$ and (ntn4 each support homophilic but not heterophilic adhesion. Scale bar: $5 \mu \mathrm{m}$ for $\boldsymbol{a}, \boldsymbol{b} ; 10 \mu \mathrm{m}$ for $\boldsymbol{c}-\boldsymbol{j}$.

fraction of retinal ganglion cells. This, although Cntns could mediate transsynaptic interactions among amacrine cells, they are less likely to mediate such interactions between amacrine and retinal ganglion cells.

Together, these observations suggest the hypothesis that Cntns could promote laminar specificity not only by mediating transsynaptic interactions, but also by mediating interactions among dendrites of a single subtype within a sublaminae. Indeed, Cntns1 and 2 were initially described as mediators of fasciculation (Chang et al., 1987; Rathjen et al., 1987; Brümmendorf et al., 1989). Although this interaction has not been emphasized in studies in vivo, it may underlie roles of Cntns in retina.

\section{Is the IgSF code for laminar specificity complete?}

Further double- and triple-labeling studies, as well as analysis at other ages, will be needed to determine precisely what fraction of retinal neurons express one of the two $s d k s$, two $d s c a m s$, and five cntns. Nonetheless, it seems that at least for amacrine cells, the vast majority of cells express at least one of these nine related IgSF genes (Fig. 12). In that cntns appear to be expressed by far more amacrine than bipolar or retinal ganglion cells, however (Fig. 4), a substantial number of cells in the latter categories may express no $s d k, d s c a m$, or cntn. Candidate markers for these cells are the four members of the NgCAM subfamily (Fig. 1). We are currently analyzing these and other IgSF members (Kim et al., 2008) in hopes of determining whether all retinal neurons can be categorized by the IgSF genes they express.

\section{References}

Andrews GL, Tanglao S, Farmer WT, Morin S, Brotman S, Berberoglu MA, Price H, Fernandez GC, Mastick GS, Charron F, Kidd T (2008) Dscam guides embryonic axons by Netrin-dependent and -independent functions. Development 135:3839-3848.

Berglund EO, Murai KK, Fredette B, Sekerková G, Marturano B, Weber L, Mugnaini E, Ranscht B (1999) Ataxia and abnormal cerebellar microorganization in mice with ablated contactin gene expression. Neuron 24:739-750.

Brümmendorf T, Wolff JM, Frank R, Rathjen FG (1989) Neural cell recognition molecule F11: homology with fibronectin type III and immunoglobulin type $\mathrm{C}$ domains. Neuron 2:1351-1361.

Buttiglione M, Revest JM, Pavlou O, Karagogeos D, Furley A, Rougon G, Faivre-Sarrailh C (1998) A functional interaction between the neuronal adhesion molecules TAG-1 and F3 modulates neurite outgrowth and fasciculation of cerebellar granule cells. J Neurosci 18:6853-6870.

Chang S, Rathjen FG, Raper JA (1987) Extension of neurites on axons is impaired by antibodies against specific neural cell surface glycoproteins. J Cell Biol 104:355-362.

Dodd J, Morton SB, Karagogeos D, Yamamoto M, Jessell TM (1988) Spatial 


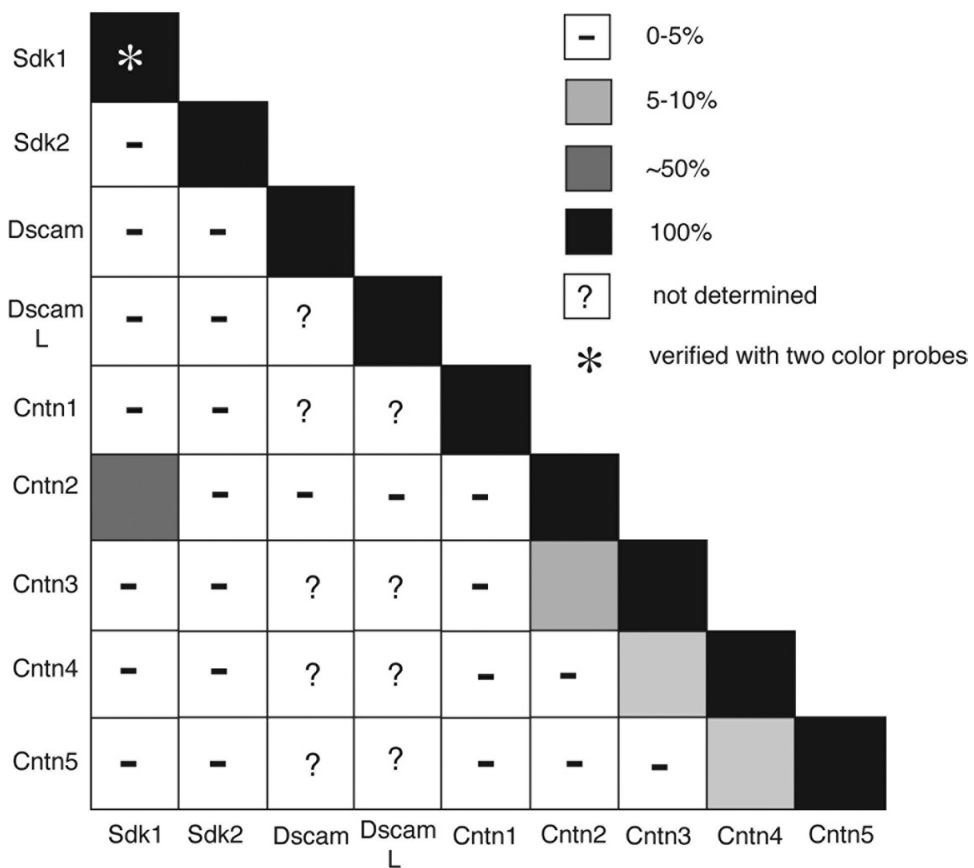

Figure 12. Largely non-overlapping expression of contactins, sidekicks, and dscams in chick retina. This table compiles data from Figures 5 (laminar distribution of (ntns in the inner plexiform layer), 6 (double-label immunostaining and in situ hybridization), and 7 (double-label in situ hybridization), and the studies by Yamagata et al. (2002) and Yamagata and Sanes (2008). Cntns and $s d k s$ are expressed by largely non-overlapping groups of retinal neurons, with the major exception being $\sim 50 \%$ coexpression of Cntn2 and Sdk1. Cells that express Sdk1, Sdk2, or Cntn2 do not coexpress either Dscam. Combined with data in Figure 4 and from the study by Yamagata and Sanes (2008), the patterns summarized here imply that at least $80 \%$ of amacrine cells express at least one of these nine IgSF genes.

regulation of axonal glycoprotein expression on subsets of embryonic spinal neurons. Neuron 1:105-116.

Drenhaus U, Morino P, Veh RW (2003) On the development of the stratification of the inner plexiform layer in the chick retina. J Comp Neurol 460:1-12.

Famiglietti EV (2005) "Small-tufted" ganglion cells and two visual systems for the detection of object motion in rabbit retina. Vis Neurosci 22:509-534.

Felsenfeld DP, Hynes MA, Skoler KM, Furley AJ, Jessell TM (1994) TAG-1 can mediate homophilic binding, but neurite outgrowth on TAG-1 requires an L1-like molecule and beta 1 integrins. Neuron 12:675-690.

Freigang J, Proba K, Leder L, Diederichs K, Sonderegger P, Welte W (2000) The crystal structure of the ligand binding module of axonin-1/TAG-1 suggests a zipper mechanism for neural cell adhesion. Cell 101: 425-433.

Gennarini G, Durbec P, Boned A, Rougon G, Goridis C (1991) Transfected F3/F11 neuronal cell surface protein mediates intercellular adhesion and promotes neurite outgrowth. Neuron 6:595-606.

Hayashi K, Kaufman L, Ross MD, Klotman PE (2005) Definition of the critical domains required for homophilic targeting of mouse sidekick molecules. FASEB J 19:614-616.

Kaneko-Goto T, Yoshihara S, Miyazaki H, Yoshihara Y (2008) BIG-2 mediates olfactory axon convergence to target glomeruli. Neuron 57:834-846

Kim IJ, Zhang Y, Yamagata M, Meister M, Sanes JR (2008) Molecular identification of a retinal cell type that responds to upward motion. Nature 452:478-482.

Kuhn TB, Stoeckli ET, Condrau MA, Rathjen FG, Sonderegger P (1991) Neurite outgrowth on immobilized axonin-1 is mediated by a heterophilic interaction with L1(G4). J Cell Biol 115:1113-1126.

Kunz S, Spirig M, Ginsburg C, Buchstaller A, Berger P, Lanz R, Rader C, Vogt L, Kunz B, Sonderegger P (1998) Neurite fasciculation mediated by complexes of axonin-1 and $\mathrm{Ng}$ cell adhesion molecule. J Cell Biol 143:1673-1690.

Lauter G, Söll I, Hauptmann G (2011) Multicolor fluorescent in situ hybridization to define abutting and overlapping gene expression in the embryonic zebrafish brain. Neural Dev 6:10.
Liu G, Li W, Wang L, Kar A, Guan KL, Rao Y, Wu JY (2009) DSCAM functions as a netrin receptor in commissural axon pathfinding. Proc Natl Acad Sci U S A 106:2951-2956.

Ly A, Nikolaev A, Suresh G, Zheng Y, TessierLavigne M, Stein E (2008) DSCAM is a netrin receptor that collaborates with DCC in mediating turning responses to netrin-1. Cell 133:1241-1254.

Masland RH (2001) The fundamental plan of the retina. Nat Neurosci 4:877-886.

Matsuoka RL, Nguyen-Ba-Charvet KT, Parray A, Badea TC, Chédotal A, Kolodkin AL (2011) Transmembrane semaphorin signalling controls laminar stratification in the mammalian retina. Nature 470:259-263.

Millar TJ, Ishimoto I, Chubb IW, Epstein ML, Johnson CD, Morgan IG (1987) Cholinergic amacrine cells of the chicken retina: a light and electron-microscope immunocytochemical study. Neuroscience 21:725-743.

Nagai T, Ibata K, Park ES, Kubota M, Mikoshiba K, Miyawaki A (2002) A variant of yellow fluorescent protein with fast and efficient maturation for cell-biological applications. Nat Biotechnol 20:87-90.

Ogawa J, Kaneko H, Masuda T, Nagata S, Hosoya H, Watanabe K (1996) Novel neural adhesion molecules in the Contactin/F3 subgroup of the immunoglobulin superfamily: isolation and characterization of $\mathrm{cDNAs}$ from rat brain. Neurosci Lett 218:173-176.

Osterfield M, Egelund R, Young LM, Flanagan JG (2008) Interaction of amyloid precursor protein with contactins and NgCAM in the retinotectal system. Development 135:1189-1199.

Pavlou O, Theodorakis K, Falk J, Kutsche M, Schachner M, Faivre-Sarrailh C Karagogeos D (2002) Analysis of interactions of the adhesion molecule TAG-1 and its domains with other immunoglobulin superfamily members. Mol Cell Neurosci 20:367-381.

Peles E, Nativ M, Lustig M, Grumet M, Schilling J, Martinez R, Plowman GD, Schlessinger J (1997) Identification of a novel contactin-associated transmembrane receptor with multiple domains implicated in proteinprotein interactions. EMBO J 16:978-988.

Poliak S, Gollan L, Martinez R, Custer A, Einheber S, Salzer JL, Trimmer JS, Shrager P, Peles E (1999) Caspr2, a new member of the neurexin superfamily, is localized at the juxtaparanodes of myelinated axons and associates with K+ channels. Neuron 24:1037-1047.

Rader C, Stoeckli ET, Ziegler U, Osterwalder T, Kunz B, Sonderegger P (1993) Cell-cell adhesion by homophilic interaction of the neuronal recognition molecule axonin-1. Eur J Biochem 215:133-141.

Ramon y Cajal S (1892) La rétine des vertébrés. La Cellule 9:119-257.

Rathjen FG, Wolff JM, Frank R, Bonhoeffer F, Rutishauser U (1987) Membrane glycoproteins involved in neurite fasciculation. J Cell Biol 104:343-353.

Roska B, Werblin F (2001) Vertical interactions across ten parallel, stacked representations in the mammalian retina. Nature 410:583-587.

Ryan MD, Drew J (1994) Foot-and-mouth-disease virus 2A oligopeptide mediated cleavage of an artificial polyprotein. EMBO J 13:928-933.

Salzer JL, Brophy PJ, Peles E (2008) Molecular domains of myelinated axons in the peripheral nervous system. Glia 56:1532-1540.

Sanes JR, Yamagata M (2009) Many paths to synaptic specificity. Annu Rev Cell Dev Biol 25:161-195.

Sanes JR, Zipursky SL (2010) Design principles of insect and vertebrate visual systems. Neuron 66:15-36.

Schreiner D, Weiner JA (2010) Combinatorial homophilic interaction between gamma-protocadherin multimers greatly expands the molecular diversity of cell adhesion. Proc Natl Acad Sci U S A 107:14893-14898.

Shimoda Y, Watanabe K (2009) Contactins: emerging key roles in the development and function of the nervous system. Cell Adh Migr 3:64-70.

Sievers F, Wilm A, Dineen D, Gibson TJ, Karplus K, Li W, Lopez R, McWil- 
liam H, Remmert M, Söding J, Thompson JD, Higgins DG (2011) Fast, scalable generation of high-quality protein multiple sequence alignments using Clustal Omega. Mol Syst Biol 7:539.

Spiegel I, Salomon D, Erne B, Schaeren-Wiemers N, Peles E (2002) Caspr3 and caspr4, two novel members of the caspr family are expressed in the nervous system and interact with PDZ domains. Mol Cell Neurosci 20:283-297.

Stoeckli ET (2010) Neural circuit formation in the cerebellum is controlled by cell adhesion molecules of the Contactin family. Cell Adh Migr 4:523-526.

Suter DM, Pollerberg GE, Buchstaller A, Giger RJ, Dreyer WJ, Sonderegger P (1995) Binding between the neural cell adhesion molecules axonin-1 and $\mathrm{Nr}-\mathrm{CAM} /$ Bravo is involved in neuron-glia interaction. J Cell Biol 131:1067-1081.

Takeda Y, Akasaka K, Lee S, Kobayashi S, Kawano H, Murayama S, Takahashi N, Hashimoto K, Kano M, Asano M, Sudo K, Iwakura Y, Watanabe K (2003) Impaired motor coordination in mice lacking neural recognition molecule NB-3 of the contactin/F3 subgroup. J Neurobiol 56:252-265.

Toyoshima M, Sakurai K, Shimazaki K, Takeda Y, Shimoda Y, Watanabe K (2009) Deficiency of neural recognition molecule NB-2 affects the development of glutamatergic auditory pathways from the ventral cochlear nucleus to the superior olivary complex in mouse. Dev Biol 336:192-200.

Traut W, Weichenhan D, Himmelbauer H, Winking H (2006) New members of the neurexin superfamily: multiple rodent homologues of the human CASPR5 gene. Mamm Genome 17:723-731.

Wässle H (2004) Parallel processing in the mammalian retina. Nat Rev Neurosci 5:747-757.

Xue Y, Honig MG (1999) Ultrastructural observations on the expression of axonin-1: implications for the fasciculation of sensory axons during axonal outgrowth into the chick hindlimb. J Comp Neurol 408:299-317.

Yamagata M, Sanes JR (2008) Dscam and Sidekick proteins direct laminaspecific synaptic connections in vertebrate retina. Nature 451:465-469.

Yamagata M, Sanes JR (2010) Synaptic localization and function of sidekick recognition molecules require MAGI scaffolding proteins. J Neurosci 30:3579-3588.

Yamagata M, Sanes JR (2012) Transgenic strategy for identifying synaptic connections in mice by fluorescence complementation (GRASP). Front Mol Neurosci 5:18.

Yamagata M, Weiner JA, Sanes JR (2002) Sidekicks: synaptic adhesion molecules that promote lamina-specific connectivity in the retina. Cell 110:649-660.

Yamagata M, Weiner JA, Dulac C, Roth KA, Sanes JR (2006) Labeled lines in the retinotectal system: markers for retinorecipient sublaminae and the retinal ganglion cell subsets that innervate them. Mol Cell Neurosci 33:296-310.

Yoshihara Y, Kawasaki M, Tani A, Tamada A, Nagata S, Kagamiyama H, Mori K (1994) BIG-1: a new TAG-1/F3-related member of the immunoglobulin superfamily with neurite outgrowth-promoting activity. Neuron 13:415-426.

Yoshihara Y, Kawasaki M, Tamada A, Nagata S, Kagamiyama H, Mori K (1995) Overlapping and differential expression of BIG-2, BIG-1, TAG-1, and F3: four members of an axon-associated cell adhesion molecule subgroup of the immunoglobulin superfamily. J Neurobiol 28:51-69.

Zuko A, Bouyain S, van der Zwaag B, Burbach JP (2011) Contactins: structural aspects in relation to developmental functions in brain disease. Adv Protein Chem Struct Biol 84:143-180. 